INPLASY

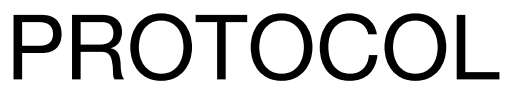

To cite: Dai et al. Tuina for functional dyspepsia: Protocol for a systematic review and meta-analysis of randomized controlled trials. Inplasy protocol 202120066. doi: 10.37766/inplasy2021.2.0066

Received: 20 February 2021

Published: 20 February 2021

Corresponding author: Jianping Liu

liujp@bucm.edu.cn

Author Affiliation:

Beijing University of Chinese Medicine

Support: NSFC(No. 81830115).

Review Stage at time of this submission: Preliminary searches.

Conflicts of interest: None declared.

\section{Tuina for functional dyspepsia: Protocol for a systematic review and meta-analysis of randomized controlled trials}

Dai, N1; Qing, YH²; Liu, XH³; Xiong, M4; Fang, M5; Li, X6; Li, DD7; Liu, HL8; Liu, JP9.

Review question / Objective: Is Chinese therapeutic massage Tuina effective for symptomatic relief of functional dyspepsia? Condition being studied: Functional dyspepsia (FD) is a functional gastrointestinal disease, which is diagnosed by one or more of the symptoms, including discomfort of fullness after a meal, discomfort of early satiety, upper middle abdominal pain and burning sensation in upper middle abdomen. The symptoms appear for at least 6 months before diagnosis, and the symptoms are more than 3 months standard. The prevalence of FD is relatively high. It is $8 \%-23 \%$ in Asia, while about $40 \%$ of adults suffer from functional dyspepsia in western countries. In addition, FD seriously reduces the work efficiency of patients, affects their quality of life, and brings a heavy economic burden. According to surveys, the cost of treating patients with FD in the United States was about 18.4 billion US dollars in 2009. Tuina, as an external treatment of Chinese medicine, has no drug side effects and can treat many diseases, especially digestive diseases. In recent years, there have been more and more clinical studies on the treatment of FD with massage. However, there is no systematic review to systematically evaluate the efficacy and safety of massage for the treatment of FD.

INPLASY registration number: This protocol was registered with the International Platform of Registered Systematic Review and Meta-Analysis Protocols (INPLASY) on 20 February 2021 and was last updated on 20 February 2021 (registration number INPLASY202120066).

\section{INTRODUCTION}

Review question / Objective: Is Chinese therapeutic massage Tuina effective for symptomatic relief of functional dyspepsia?
Condition being studied: Functional dyspepsia (FD) is a functional gastrointestinal disease, which is diagnosed by one or more of the symptoms, including discomfort of fullness 
after a meal, discomfort of early satiety, upper middle abdominal pain and burning sensation in upper middle abdomen. The symptoms appear for at least 6 months before diagnosis, and the symptoms are more than 3 months standard. The prevalence of FD is relatively high. It is $8 \%-23 \%$ in Asia, while about $40 \%$ of adults suffer from functional dyspepsia in western countries. In addition, FD seriously reduces the work efficiency of patients, affects their quality of life, and brings a heavy economic burden. According to surveys, the cost of treating patients with FD in the United States was about $\mathbf{1 8 . 4}$ billion US dollars in 2009. Tuina, as an external treatment of Chinese medicine, has no drug side effects and can treat many diseases, especially digestive diseases. In recent years, there have been more and more clinical studies on the treatment of FD with massage. However, there is no systematic review to systematically evaluate the efficacy and safety of massage for the treatment of FD.

\section{METHODS}

Search strategy: We will search the following electronic databases from their inception dates to January 31st, 2021 for potentially relevant studies, and languages will be restricted to Chinese and English only. China National Knowledge Infrastructure(CNKI); Wangfang Database; Chinese Science Technology Journal Database (VIP); SinoMed; Cochrange Library, including the trials registers of the Cochrane Gut Review Group and the Cochrane Central Register of Controlled Trials(CENTRAL); Pubmed; Embase. We will also search the following trials registers: International Standard Randomized Controlled Trial Number Register (www.controlled-trials.com); US National Institutes of Health Ongoing Trials Register (www.ClinicalTrials.gov); World Health Organization International Clinical Trials Registry (www.who.int/ictrp/en/). In addition, the reference lists of all identified studies will also be searched to find any further relevant trials for inclusion. Search strategy: \#1 Tuina [Title/Abstract] OR Tui na OR [Title/Abstract]massage [Title/Abstract] OR rubbing abdomen [Title/Abstract] OR chiropractic [Title/Abstract] OR technique [Title/Abstract] OR spinal manipulation [Title/Abstract] \#2 epigastric pain syndrome [Title/Abstract] OR postprandial distress syndrome [Title/Abstract] OR dyspepsia[MeSH Terms] OR functional dyspepsia [Title/Abstract] OR indigestion [Title/Abstract] OR indigestive [Title/ Abstract] \#3 a RCT filter \#4 \#1AND \#2 AND \#3.

Participant or population: We will include researches involving people with functional dyspepsia according to Rome (特),(監),(企) or (協) diagnostic criteria, of both genders and any age. Patients with other types of diseases, such as severe cardiac dysfunction, severe hepatic dysfunction, severe kidney dysfunction, endocrine disease, cholecystitis, pancreatitis, and peptic ulcer, will be excluded.

Intervention: We will include all types of Tuina manipulation, without limitations on the manipulation technique used, the number of Tuina sessions administered, or the duration over which Tuina is used. The Tuina treatment will not be combined other traditional Chinese therapy; however, it may be used alone, or in combination with conventional medical treatment, such as acid suppression agents, prokinetics agents, $H$. Pylori eradication, and Histamine 2-receptor antagonists (H2RAs), and so on.

Comparator: The control intervention will include: no treatment, conventional medical treatment, such as acid suppression agents, prokinetics agents, $\mathrm{H}$. Pylori eradication, and H2RAs, and so on. Co-invention will be allowed as long as all arms of the randomized allocation received the same co-invention.

Study designs to be included: Randomized, parallel clinical trials will be included irrespective of blinding, publication status and language. Randomized cross-over trials will be included only if the trial reported a wash-out period to eliminate any carry-over effect. Quasi-randomized 
trials and controlled clinical trials without randomization will be excluded.

Eligibility criteria: Participants/population: We will include researches involving people with functional dyspepsia according to Rome (特,,(監),(企) or (協) diagnostic criteria, of both genders and any age. Patients with other types of diseases, such as severe cardiac dysfunction, severe hepatic dysfunction, severe kidney dysfunction, endocrine disease, cholecystitis, pancreatitis, and peptic ulcer, will be excluded. Interventions: We will include all types of Tuina manipulation, without limitations on the manipulation technique used, the number of Tuina sessions administered, or the duration over which Tuina is used. The Tuina treatment will not be combined other traditional Chinese therapy; however, it may be used alone, or in combination with conventional medical treatment, such as acid suppression agents, prokinetics agents, $H$. Pylori eradication, and Histamine 2-receptor antagonists (H2RAs), and so on. Control: The control intervention will include: no treatment, conventional medical treatment, such as acid suppression agents, prokinetics agents, H. Pylori eradication, and H2RAs, and so on. Co-invention will be allowed as long as all arms of the randomized allocation received the same co-invention. Types of study to be included: Randomized, parallel clinical trials will be included irrespective of blinding, publication status and language. Randomized cross-over trials will be included only if the trial reported a washout period to eliminate any carry-over effect. Quasi-randomized trials and controlled clinical trials without randomization will be excluded. Main outcomes: The main outcome measures sought at the end of treatment and at maximal follow-up after completion of the treatment will be global improvement of symptoms (patient-reported and/or clinician-evaluated) and quality of life. Additional outcomes: (1)Number of recurrent episodes; (2)Subtypes of predominant symptom: discomfort of fullness after a meal, discomfort of early satiety, upper middle abdominal pain and burning sensation in upper middle abdomen; (3)Cost-effectiveness; (4)Number and type of adverse events. Two types of adverse events were analyzed, serious adverse events and adverse events not considered serious. The serious adverse events were any untoward medical occurrence that resulted in death, was lifethreatening, required hospitalization or prolongation of hospitalization, resulted in persistent or significant disability, are congenital anomalies/birth defects or events that may jeopardize the patient or required intervention to prevent one of the former serious adverse events. All other adverse events will be considered nonserious. (5)Gastrointestinal hormones. We will observe the changes of gastrointestinal hormones, including ghrelin, motlin, gastrin, etc.. in functional dyspepsia patients' blood after treatment.

Information sources: We will search the following electronic databases from their inception dates to January 31st, 2021 for potentially relevant studies, and languages will be restricted to Chinese and English only. China National Knowledge Infrastructure(CNKI); Wangfang Database; Chinese Science Technology Journal Database(VIP); SinoMed; Cochrane Library, including the trials registers of the Cochrane Gut Review Group and the Cochrane Central Register of Controlled Trials(CENTRAL); Pubmed; Embase. We will also search the following trials registers: International Standard Randomized Controlled Trial Number Register (www.controlled-trials.com); US National Institutes of Health Ongoing Trials Register (www.ClinicalTrials.gov); World Health Organization International Clinical Trials Registry (www.who.int/ictrp/en/). In addition, the reference lists of all identified studies will also be searched to find any further relevant trials for inclusion.

Main outcome(s): The main outcome measures sought at the end of treatment and at maximal follow-up after completion of the treatment will be global improvement of symptoms (patient-reported and/or clinician-evaluated) and quality of life. 
Additional outcome(s): (1)Number of recurrent episodes; (2)Subtypes of predominant symptom: discomfort of fullness after a meal, discomfort of early satiety, upper middle abdominal pain and burning sensation in upper middle abdomen; (3)Cost-effectiveness; (4)Number and type of adverse events. Two types of adverse events were analyzed, serious adverse events and adverse events not considered serious. The serious adverse events were any untoward medical occurrence that resulted in death, was lifethreatening, required hospitalization or prolongation of hospitalization, resulted in persistent or significant disability, are congenital anomalies/birth defects or events that may jeopardize the patient or required intervention to prevent one of the former serious adverse events. All other adverse events will be considered nonserious. (5)Gastrointestinal hormones. We will observe the changes of gastrointestinal hormones, including ghrelin, motlin, gastrin, etc.. in functional dyspepsia patients' blood after treatment.

Data management: Four review authors (M Fang, QY He, XH Liu and M Xiong) will independently extract the following study characteristics and outcome data from included studies by using standard data extraction forms and check them. Data from trials in duplicate will be included only once. Any disagreement will be resolved through discussion or by consultation with a third review author (JP Liu). In addition, if any information in a given study is missing or unclear, we will try to contact the authors of the study for more specific information. Methods: study design, total duration of study, number of study centers and location, study setting, withdrawals, date of study. Participants: number, mean age, age range, gender, severity of condition, diagnostic criteria, inclusion criteria, exclusion criteria. Interventions: name of intervention, schedule, comparison, concomitant medications, excluded medications. Outcomes: primary and secondary outcomes specified and collected, time points reported. Notes: funding for trial, notable conflicts of interest of trial authors. One review author
(N Dai) will enter the data from the data extraction forms into Review Manager (RevMan5.3, 2014), and another author ( $X$ Li) will check it.

Quality assessment / Risk of bias analysis: o authors (DD $L i$ and $H L$ Liu) will independently assess risk of bias for each study using the Cochrane 'Risk of bias' assessment tool (Higgins 2011). Any disagreements will be resolved by consensus or by consultation with a third review author ( $\mathrm{JI}$ Liu). We will assess the following domains. Sequence generation (selection bias); Allocation concealment (selection bias); Blinding of participants and personnel (performance bias); Blinding of outcome assessment (detection bias); Incomplete outcome data (attrition bias); Selective outcome reporting (reporting bias); Other potential sources of bias (e.g. baseline imbalance). We will assess each potential source of bias as 'high,' 'low,' or 'unclear' as described in the Cochrane Handbook for Systematic Reviews of Interventions (Higgins 2011). We will present a 'Risk of bias' summary figure and a 'Risk of bias' graph to illustrate these findings.

Strategy of data synthesis: Each type of Tuina manipulation will be compared with no treatment or conventional medical treatment individually regardless of route of administration, does or preparation. We will undertake meta-analysis only if we consider that the participants, interventions, comparisons, and outcome assessment are similar enough to ensure that an answer would be clinically meaningful. Otherwise, we will present a qualitative synthesis to describe the results across the included studies. Dichotomous data will be presented as relative risk (RR) and continuous outcomes as mean difference (MD), both with $95 \%$ confidence intervals ( $\mathrm{Cl}$ ). Analyses will be performed by intention-to-treat where possible. For dichotomous outcomes, patients with incomplete or missing data will be included in a sensitivity analysis by counting them as treatment failures to explore the possible effect of loss to follow-up on the findings ('worst-case' scenario). 
Subgroup analysis: If sufficient data are available, we will carry out the subgroup analyses to reveal any effect that might explain any heterogeneity, including Tuina manipulation, types of FD (epigastric pain syndrome and postprandial distress syndrome), diagnostic criteria, participants ages and treatment duration (less than four weeks versus four weeks and versus greater than four weeks). We will assess differences between subgroups with the 12 statistic to test for subgroup interactions.

Sensitivity analysis: We will perform sensitivity analyses by different summary statistics (RR versus odds ratio (OR)) and meta-analytic models (fixed effect versus random-effects), to assess the robustness of our results.

Language: Languages will be restricted to Chinese and English only.

Country(ies) involved: China.

Keywords: Tuina; functional dyspepsia; systematic review; meta-analysis.

Contributions of each author:

Author 1 - Ning Dai - The author drafted the manuscript, selected the included studies and analyzed the data.

Email: daining0719@bucm.edu.cn

Author 2 - Qingyun $\mathrm{He}$ - The author was responsible for selecting the trials of inclusion and extracting data.

Email: hqyheather@126.com

Author 3 - Xuehan Liu - The author contributed to the data extraction.

Email: xuehan_liu@foxmail.com

Author 4 - Min Xiong - The author contributed to the data extraction.

Email: 929456424@qq.com

Author 5 - Min Fang - The author contributed to the data extraction.

Email: fangmin353@163.com

Author 6 - Xun Li - The author was responsible for searching references.

Email: tina000341@163.com

Author 7 - Duoduo Li - The author contributed to assess risk bias in included studies.

Email: tarako@163.com
Author 8 - Hongliang Liu - The author contributed to assess risk bias in included studies.

Email: zhuimeng969@163.com

Author 9 - Jianping Liu - The author contributed to design the review and deal with disagreement.

Email: liujp@bucm.edu.cn 